\title{
LAS DIMENSIONES RELACIONALES Y SIMBÓLICAS DE LOS SISTEMAS EDUCATIVOS: HIPÓTESIS PARA EL CASO CHILENO
}

\author{
RELATIONAL AND SYMBOLIC DIMENSIONS OF EDUCATIONAL \\ SYSTEMS: A HYPOTHESIS FOR THE CHILEAN CASE
}

\section{JAVIER CORVALÁN ${ }^{2}$}

\section{RESUMEN}

Utilizando algunos de los conceptos centrales de la teoría sociológica de Pierre Bourdieu, se conceptualizan los sistemas educativos actuales y el chileno en particular como espacios sociales en los cuales operan distinciones simbólicas sobre la base de la existencia relacional de sus componentes. Se analizan aspectos particulares de tales distinciones para el caso chileno y se sostiene que los análisis político-públicos de tal sistema no lo han concebido de esa manera sino que, por el contrario, lo han estudiado como unidades no relacionadas.

Palabras clave: Sistema educativo chileno, Bourdieu, campo educativo.

\section{ABSTRACT}

By using some of the central concepts of Bourdieu's sociological theory, current educational systems are conceptualized, and the Chilean case in particular, as social spaces where different symbolic distinctions are produced on the basis of the relational existence of its components. Particular aspects of such distinctions are analysed for the Chilean case and it is maintained that the political-public analysis of such a system has not been conceived in this way; on the contrary, the system has been studied as containing non related units.

Keywords: Chilean educational system, Bourdieu, educational field.

Recibido: 15.07.12. Aceptado: 10.06.13.

${ }^{1}$ Investigación financiada por el Centro de Estudios de Políticas y Prácticas en Educación (CEPPE), Proyecto CIE01-CONICYT como parte de la línea Políticas Educativas, sublínea Mercados educativos en Chile.

${ }^{2}$ Doctor en sociología. Director del Magíster en Política Educativa de la Facultad de Educación de la Universidad Alberto Hurtado. Santiago, Chile. E-mail: jcorvala@cide.cl 
AS PÁGINAS SIGUIENTES se concentran en la aplicación de una serie de conceptos propios de la teorización de Pierre Bourdieu con el fin de dar cuenta de ciertas características, algunas de ellas paradojales, del sistema educacional chileno. La hipótesis central de este trabajo parte de una de las constataciones básicas del debate sociológico-educativo a partir de Bourdieu y que se refiere a que todo sistema educativo en las sociedades contemporáneas es un espacio de distinción simbólica, como consecuencia, principalmente, de los procesos de masificación y de diversificación de la oferta educativa, propios de la mayor parte de las sociedades occidentales desde mediados del siglo XX.

Con esta afirmación seguimos muy de cerca la teoría de nuestro autor, entendiendo que el sistema educativo es, en términos analíticos, un campo, es decir, un espacio social de lucha entre agentes por legitimidades y con ello, de disputa por el control del campo mismo ${ }^{3}$. Desde esta perspectiva, un sistema educativo puede ser entendido como un espacio simbólico que, como tal, construye distinciones, diferencias, distancias y proximidades entre los agentes que lo integran y produce, además, los objetos de disputa entre estos agentes, así como también el valor de los capitales requeridos para vencer o perder en esa refriega permanente. Las distinciones y diferenciaciones espaciales y simbólicas entre los agentes, en este enfoque teórico, están compuestos por discursos y prácticas, que ponen en juego determinados capitales y que, por lo mismo, encierran valoraciones, controles y subordinaciones.

Junto a esta consideración nos parece de importancia para el tema de este articulo aludir a dos conceptos algo menos conocidos de nuestro autor: doxa e illusio. La doxa, en la teorización de Bourdieu, se refiere a aquello que siendo arbitrario se considera evidente ${ }^{4}$. Así, la doxa constituye una suerte de conciencia común que en particular se asume en un determinado campo. Por su parte, la illusio se refiere a aquello que determina el interés por la disputa de lo que está en juego ${ }^{5}$. La illusio es la creencia compartida que aquello que se discute tiene valor, es "la condición indiscutida de la

${ }^{3}$ Los campos son definidos por Bourdieu como "universos sociales que tienen una ley fundamental, un nomos independiente de aquel de otros universos que son auto-nomos que evalúan lo que hacen, los desafíos que en ellos se producen según principios y criterios irreductibles a aquellos de otros universos" (Bourdieu, 2007, p. 19).

4 “... doxa es una ortodoxia, una visión asumida, dominante, que solo al cabo de las luchas contra las visiones contrarias ha conseguido imponerse” (Bourdieu, 2007, p. 120).

5 "la illusio es el hecho de estar metido en el juego, cogido por el juego, de creer que el juego merece la pena, que vale la pena jugar...es decir, el hecho de considerar que un juego social es importante, que lo que ocurre en él importa a quienes están dentro” (Bourdieu, 2007, p. 141). 
discusión" (Bourdieu, 1997, p. 122); la illusio hace que la participación de los agentes en el campo sea un juego, pero un juego interesante y a veces dramático de ser jugado.

Considerar al sistema educativo como campo equivale a señalarlo como espacio simbólico y por tanto provisto también de una determinada doxa e illusio, un conjunto de verdades impuestas y válidas como realidades absolutas en su interior. Junto a ello es importante señalar algo que está en la base de todo espacio simbólico: su naturaleza relacional, es decir, que sus constituyentes toman valor e incluso existencia a partir de la ubicación que tengan en él y que tal ubicación no es absoluta ni intrínseca, sino relativa a los demás elementos.

Nos interesa conceptualizar el sistema educativo chileno de esta manera, en contraposición a como se ha llevado a cabo la mayor parte de sus análisis en las últimas dos décadas, en especial en lo relacionado con la temática recurrente de la inequidad educacional, la que ha tendido a definirse con ausencia de una comprensión de este sistema en términos tanto relacionales como simbólicos. Mirado desde los aportes teóricos de Bourdieu, lo anterior constituye una fuerte limitación conceptual del debate socioeducativo chileno que se expresa, de manera muy evidente, en la afirmación de que el sistema escolar, refiriéndonos en particular a aquel que se forma después de las transformaciones estructurales de los años $80^{6}$ es, por sobre todo, un mercado, afirmándose de paso una supuesta novedad o descubrimiento respecto de tal sistema (ver, por ejemplo, OCDE, 2004).

Por supuesto, no es nuestra intención afirmar que el sistema educativo chileno no tiene un funcionamiento de mercado sino que, de acuerdo a lo que nos señala el mismo Bourdieu, un mercado, más allá de la manera como lo conceptualiza la economía, es siempre un espacio simbólico (Bourdieu, 2000) y que si bien es cierto como mercado es analizable en tanto escenario de transacciones objetivadas, no es menos cierto que es, a la vez, un escenario de distinciones que operan tanto en la objetividad como en la subjetividad de los agentes que en él existen.

\footnotetext{
${ }^{6}$ Se trató de una trasformación estructural del sistema educacional chileno, realizada en un contexto no democrático y en la que, al mismo tiempo que se traspasaron las escuelas fiscales a los municipios, se incentivó el aumento de establecimientos educacionales privados y a la vez subvencionados por el Estado. En la base de estas transformaciones se encuentra un cambio también en la asignación de recursos, haciéndose competitiva a partir de una subvención otorgada por la asistencia de cada niño a una escuela determinada. Se trató entonces de la creación de un cuasi-mercado educativo propiamente tal, aun cuando el elemento de precio a los usuarios estuvo ausente hasta los años 90, con la introducción del mecanismo de financiamiento compartido (ver al respecto más adelante en este mismo artículo).
} 
Más allá de esta insuficiencia conceptual importa también relativizar, a la luz de los aportes de Bourdieu, la supuesta originalidad en la conceptualización del sistema educativo como un mercado. En realidad, uno de los grandes aportes aunque tal vez indirectos de Bourdieu, es no solo respecto del contenido simbólico de todo mercado, sino también la constatación de que todo sistema educativo constituye, en muchos de sus aspectos, un mercado, y que ello no es privativo de la organización liberal y/o mercantilizada del mismo, como se observa en el caso chileno.

En efecto, cuando afirmamos que los sistemas educacionales contemporáneos constituyen en mayor o menor medida escenarios de mercado independientemente de si en ellos existe o no la monetarización y el precio al cliente, estamos haciendo una constatación sobre la cual probablemente no estarían de acuerdo muchos economistas, pero que se refiere a la manera en que los procesos educacionales están organizados en las sociedades contemporáneas a partir de dinámicas competitivas entre individuos y/o instituciones, todo ello producto de la función de selección asignada a las instituciones educacionales ${ }^{7}$. En este punto es importante recordar, a partir de los postulados más básicos de la sociología económica (Fligstein y Dauter, 2007) y refrendados de manera indirecta por el mismo Bourdieu (2000), que un mercado es un escenario en el cual existe uno o algunos recursos en disputa y que para llevar a cabo tal disputa, los agentes deben desarrollar acción competitiva, cuyo resultado final los diferenciará.

Si se asume esta definición entonces se deberá reconocer que el escenario de la sala de clases y del marco institucional y regulador escolar en el cual ella se enmarca constituye un mercado por cuanto los alumnos disputan recursos (resultados de evaluación, aprobaciones o desaprobaciones) mediante acciones competitivas de resultados individuales y diferenciadores. En consecuencia, parece importante asumir el sistema y la dinámica educacional al menos parcialmente como un mercado, independientemente de su nivel de monetarización de las relaciones establecidas, al mismo tiempo que se debe tener en cuenta el carácter simbólico de todo escenario mercantil.

Con todo, no está demás recordar que si todo sistema educativo es en alguna medida un mercado, que si, además, todo mercado es también un escenario fuertemente simbolizado y que si, finalmente, el sistema educati-

\footnotetext{
${ }^{7}$ La función de selección, propuesta como tal por los análisis estructural-funcionalistas de la educación, se refiere al proceso mediante el cual las instituciones educacionales van clasificando a los individuos de acuerdo a sus desempeños académicos entregando así señales a otras instituciones educativas y sociales, particularmente al mercado laboral.
} 
vo chileno es con seguridad el más mercantil de todos los sistemas educativos, la consecuencia será que su nivel de simbolización está fuertemente agudizado. Para refrendar esta afirmación basta aludir a dos construcciones objetivo-simbólicas que están en la base del sistema educativo chileno y que, de paso, son casi únicas en el mundo: el $\mathrm{SIMCE}^{8}$ y el financiamiento compartido 9 . Ambos elementos son, a su vez, una genuina expresión del desarrollo de un mercado educacional, por un lado, el SIMCE, en tanto señal clave para incrementar y objetivar la competencia escolar y por otro el financiamiento compartido, como incorporación del cobro al cliente en la provisión educativa.

El SIMCE, referido exclusivamente para la temática de este artículo, es un elemento de distinción simbólica agregado al campo educativo. Esto, por cuanto aun sin SIMCE parece evidente que todo sistema educacional constituye jerarquías entre las escuelas, distinguiendo aquellas que son más o que son menos en algo ${ }^{10}$, importando poco si ese elemento es objetivamente real o no, ya que lo relevante es que los agentes actúan como si tal(es) elemento(s) fuesen objetivamente reales. EL SIMCE incorpora una objetividad externa a esta jerarquización (el puntaje del establecimiento) que, en la medida en que sea interiorizada y valorizada por los agentes, generará distinciones y jerarquizaciones, ya sean nuevas o complementarias, a las ya existentes.

Por su parte, el financiamiento compartido incorpora el elemento precio en las escuelas, lo cual, al igual que el SIMCE, es un mensaje objetivado, pero en este caso lo es del valor monetario del servicio educativo en cada escuela. La simbolización del precio en toda sociedad moderna y en particular en una con extensas áreas de mercado como es Chile, jerarquiza las realidades monetarizadas y les asocia otros aspectos, como por ejemplo, la calidad del bien con el monto de su valorización.

Lo anterior, curiosamente, atenta contra objetivos del propio sistema educativo, definido incluso como estructura mercantil. Un par de ejemplos al respecto: todo sistema educativo explícitamente organizado desde una

${ }^{8}$ Sistema de medición de la calidad de la educación, prueba estandarizada aplicada de manera censal cada año en algunos niveles del sistema educacional chileno (www.simce.cl).

${ }^{9}$ El financiamiento compartido es una innovación en el sistema educativo chileno, establecida en la primera mitad de los años 90 y que consiste en la posibilidad de que establecimientos subvencionados cobren una colegiatura (con límite) a las familias. Hasta la actualidad se excluye de este mecanismo a la enseñanza básica municipal (pública).

${ }^{10}$ Aludiendo a esta jerarquización Bourdieu en uno de sus libros clásicos construye, por ejemplo, el campo de las Grandes Escuelas, instituciones de educación superior en Francia en los años 80 , señalando la jerarquía de las mismas con independencia del cobro a los alumnos (Bourdieu, 1989). 
perspectiva de mercado, es decir con variables visibles de monetarización y de competencia, tenderá a hipertrofiar las distinciones de elección sobre todo cuando en ellas se introduce la variable monetaria, como ocurre en el sistema subvencionado chileno con la adopción del mecanismo de financiamiento compartido. Se observa entonces con mucha fuerza lo que todos sabemos que ocurre en Chile y es que la elección escolar no está necesariamente orientada por la calidad académica, entendida ésta en términos objetivos y unívocos, de los establecimientos sino por elementos que dicen relación con el "estatus", es decir, un conjunto de creaciones simbólicas asumidas por la dinámica del campo y ejercida por los agentes como una manera de obtener distinción y exclusividad (y en consecuencia inclusión y exclusión) y no necesariamente calidad educacional, si por esta última se entiende a los niveles de aprendizaje ${ }^{11}$.

En concreto y a manera de ejemplo: ¿por qué las familias eligen un determinado establecimiento?; ¿por qué por ejemplo hay familias que optan por un colegio particular privado de 270 puntos SIMCE ${ }^{12}$ pagando una costosa mensualidad, pudiendo optar a colegios subvencionados de SIMCE igual o mayor? Bourdieu nos da una clave de respuesta a este fenómeno y que atenta contra el funcionamiento eficaz del "mercado educativo". Esta respuesta consiste en entender la elección escolar como un proceso de distinción simbólica al interior de un espacio simbólico: al elegir o des-elegir un establecimiento se está operando con un conjunto de representaciones de sí mismo y de los demás y con una representación del espacio social en el que se inserta el establecimiento. A su vez, el concepto mismo de elección está cuestionado, en su sentido plenamente liberal, por el enfoque teórico de este artículo, por cuanto si por elección se alude a preferencia esta última se construye desde el lugar que se ocupa en el campo simbólico: la elección no es tanto un producto del individuo sino de la estructura encarnada en el individuo, lo que implica hacer referencia a otro de los conceptos centrales de este enfoque teórico, el habitus. Lejos de esto se encuentra la idea de la elección racional que supone un "mercado puro", es decir escasamente simbólico, en el cual la elección está orientada por la racionalidad de logro SIMCE y la disposición al pago creciente por el mismo.

${ }^{11}$ En encuestas aplicadas a actores del sistema educativo por la Facultad de Educación de la Universidad Alberto Hurtado durante la década del 2000 se observa que cerca de la mitad de los padres de los alumnos no le adjudican mayor importancia al puntaje SIMCE del establecimiento. Ver http://www.cide.cl/htm/encuestas.htm

${ }^{12}$ El SIMCE tiene puntajes que van entre 200 y 350 puntos, aproximadamente. 
El tema se complica aún o más bien adquiere cada vez mayor simbolización con la introducción del elemento monetario para lo cual usaremos un segundo ejemplo. En una investigación que llevamos a cabo hace algunos años respecto, precisamente, del financiamiento compartido (Corvalán 2003), constatamos que en colegios que habían pasado de gratuitos a pagados había apoderados muy contentos de lo mismo, no por el hecho de un eventual mejoramiento en la calidad académica del establecimiento sino por dos consecuencias: i) la "depuración" del establecimiento mediante la salida de aquellos que no pueden pagar la nueva colegiatura; y ii) el hecho de poder decir, de ahora en adelante, que la familia envía a sus hijos a un colegio pagado. En la combinación de ambos elementos vemos que la búsqueda de distinción conlleva un resultado de exclusión.

Consistentemente con lo anterior existe otro aspecto de gran importancia en la sociología de Bourdieu al momento de analizar la realidad educacional y que a la vez es uno de los fundamentos epistemológicos y metodológicos de sus análisis. Nos referimos a la comprensión de la realidad social como un fenómeno relacional y por lo tanto no sustancial. Sin embargo y de manera opuesta a este postulado bourdiano, la mayor parte del pensamiento socioeducativo chileno reciente ha conceptualizado la realidad educacional en términos sustanciales, aun cuando su dinámica como conjunto, en particular su reproducción, se explica relacionalmente. Solo un ejemplo al respecto: los procesos de reforma educacional basados en una lógica de mejoramiento de escuelas, fuertemente en boga en las últimas dos décadas en Chile se sustentan en una lógica de pensamiento sustancial y no relacional en la cual se postula que "mejorando" una a una las escuelas más deficitarias del país (entendiendo por tal al aumento de los resultados de aprendizaje de los alumnos) es posible llegar a un sistema óptimo y equilibrado, equitativo en palabras políticas, lo que está conceptualmente bien representado por el llamado movimiento de investigación y mejora escolar (para una visión crítica al respecto ver Carrasco, 2008). La crítica que nuestro marco teórico central aplica a este tipo de postulado es que el sistema educacional es una abstracción relacional, es decir, un conjunto de instituciones (escuelas) que por una parte integran, rechazan y/o expulsan alumnos así como también los captan de otras escuelas, a la vez que estas escuelas son interpretadas y simbolizadas por las familias-clientes de tal sistema. Por ello, el mejoramiento de una escuela se consigue no pocas veces a partir del desmejoramiento de otras mediante la atracción de alumnos con mayor capital cultural y de expulsión o rechazo al ingreso de quienes tienen menor capital cultural. En contrapartida es necesario proponer 
que el mejoramiento de escuelas, como realidad conceptual y empírica, es siempre un elemento intrínsecamente relacional: se es mejor o peor que otras e incluso también respecto de sí misma y con ello tal mejoramiento es una construcción de jerarquía simbólica y el propio movimiento y/o el de los otros concurrentes del campo en cuestión genera un reordenamiento de cada uno de los agentes del mismo. Teóricamente es esto lo que Bourdieu señala en términos de que lo real es siempre sinónimo de lo relacional (Bourdieu, 2007, p. 13). Nuestro postulado central a este respecto es que la política educativa ha entendido como fenómenos absolutos a realidades relacionales.

Teniendo en cuenta todas estas consideraciones preliminares proponemos ahora observar el sistema educativo chileno a la luz del pensamiento de Bourdieu, en al menos tres aspectos específicos. El primero es el mismo que llamó la atención poderosamente a nuestro autor en Francia hace algunas décadas y es el de la contradicción flagrante que representa el sistema educacional en una sociedad moderna y democrática y que se expresa en el título de su famoso libro, La reproducción (Bourdieu, 1970) y las consecuencias empíricas de tal fenómeno en otros ámbitos de la sociedad. Utilizando un enunciado que el mismo Bourdieu propone (Bourdieu, 2007, p. $34)$, nos referiremos brevemente a tal contradicción en términos del demonio de Maxwell.

Un siguiente aspecto del pensamiento de Bourdieu que resulta, a nuestro juicio, interesante para explicar ciertos aspectos del sistema educativo chileno dice relación con la formación del campo educativo (Bourdieu, 1979), para seguir haciendo referencia a uno de los conceptos centrales de la sociología de nuestro autor. Si se aceptan los supuestos fundamentales de tal teorización, estamos hablando de un espacio social en el cual un conjunto de agentes, provistos de capitales económicos, culturales y simbólicos desiguales, se disputan sentidos y definiciones básicas de lo educativo. Tales agentes se jerarquizan y se subordinan generando, por lo mismo, inclusiones y exclusiones. Tal espacio está, además, en permanente movimiento y precisamente, uno de los aspectos que lo caracteriza es su dinámica antes que su estática. Nos parece en consecuencia importante preguntarse por las actuales illusio y doxa de este campo. Volveremos más adelante sobre este punto.

Un último aspecto a tratar se refiere al título de otro de los libros más famosos de Bourdieu, La distinción (Bourdieu, 1979), y es que todo espacio simbólico que se recrea y amplía por efectos de la dinámica que se le imprime es, por naturaleza, un escenario en el cual los grupos de mayor 
poder pueden abandonar prácticas y/o espacios sociales para llevar a cabo una producción simbólica que pueda restablecer constantemente las distancias sociales con otros grupos. En este acto, propio pero no exclusivo de las sociedades contemporáneas, los capitales simbólicos se avalúan y se devalúan y con ello se devalúan también los logros del proceso de democratización y del acceso a bienes materiales y simbólicos de los sectores que han experimentado movilidad social ascendente. A la vez, producto de este mismo proceso, los sectores dominantes que inician su carrera por distinciones, por lo mismo abandonan prácticas que les han sido propias por largo tiempo. Un ejemplo al respecto son las prácticas deportivas ya que ellas comienzan en términos institucionalizados como un monopolio de los sectores medios y altos de la sociedad, pero en algunos casos los procesos de desarrollo y de aumento del nivel de vida, particularmente durante el siglo XX, hacen que tales prácticas sean asumidas por los restantes sectores sociales. A este respecto el fútbol es uno de las mejores evidencias ya que su popularización hizo que parte de las élites se desplazaran buscando la distinción en otras prácticas deportivas que requieren, por lo general, de capital económico elevado (ejemplos clásicos son el golf y el polo). Proponemos hacer uso, más adelante, de este concepto de desplazamiento para dar cuenta de fenómenos similares en el sistema escolar chileno.

\section{EL DEMONIO DE MAXWELL}

En uno de sus libros (Bourdieu, 2007) y a propósito del sistema educacional, Bourdieu hace referencia a esta expresión, que no le es propia sino que pertenece al físico James Maxwell quien, al referirse a la segunda ley de la termodinámica y que impide que entre dos cuerpos a diferente temperatura sea posible transmitir el calor del cuerpo frío al cuerpo caliente. En este fenómeno natural, imagina Maxwell, interviene un demonio que empieza a seleccionar las moléculas más rápidas y a desechar las más lentas. Bourdieu usa esta mitología moderna para referirse al actual sistema escolar. Con todo, el tema en cuestión es inabordable e incomprensible si no se viaja a los principios fundacionales de los sistemas educacionales modernos y constitutivos no sólo del campo educacional sino también de su illusio y su doxa y si no se contrasta a tales principios fundacionales con los análisis empíricos del sistema escolar chileno que quedarían bien reflejados bajo el título de La Reproducción. Como lo señala el mismo Bourdieu y otros sociólogos franceses (destacamos particularmente aquí a Dubet y Martuc- 
celli, 1998), la creación de la escuela y de los sistemas educacionales modernos obedece en gran parte a un ideario de igualdad (igualdad de oportunidades diríamos hoy en día) ${ }^{13}$.

En efecto, si por algún motivo la sociedad acepta que la instrucción especializada no sólo sea para las élites, es porque se reconoce y legitima el rechazo a la reproducción social como fundamento de una sociedad democrática. Es por ello que, tal vez de todas las instituciones modernas, la escuela y el sistema que la alberga sean los más contradictorios de todos: instituciones creadas para generar igualdad social mediante la instrucción universal terminan no solo reproduciendo la desigualdad sino, incluso, legitimándola. No fue para reproducir las diferencias que la escuela pública y universal se hizo (Dubet y Martuccelli, 1998), pero desde Bourdieu se reconoce que, en alguna medida y por mecanismos sutiles y complejos, esto a veces ocurre. En este punto surgen algunas preguntas a la luz del legado de Bourdieu: ¿en qué momento entonces se perdió este relato original?; ¿se ha perdido efectivamente?; ¿ está la sociedad chilena en proceso de generar una nueva doxa, es decir, una nueva narrativa que entienda la legitimidad del sistema educacional de una manera distinta, como creadora de diferencias e incluso de desigualdades legítimas?

\section{LA NUEVA ILLUSIO}

No deja de ser sorprendente la profunda transformación del campo educativo chileno en las últimas décadas. De una estructura en la que existía un número bastante reducido de agentes participantes y claramente jerarquizados a partir del protagonismo del Estado y de la iglesia católica, se llega hoy en día a un escenario de disputa -como en todo campo- en el que participa una enorme cantidad de agentes y en el que por lo tanto se agudizan tales procesos de disputa, de alianzas y de jerarquizaciones. Dentro de esta

\footnotetext{
${ }^{13}$ A manera de ilustración, en el caso de Chile el influyente libro de los hermanos Amunátegui a mediados del siglo XIX (que fue un estudio encargado y premiado por el Gobierno de Chile) da cuenta en varios párrafos de este ideal de provisión y generación de igualdad social a partir de la educación, por ejemplo cuando se refiere a que la educación pública debe ser gratuita porque: "Para nosotros la elección no es dudosa: estamos por la instrucción gratuita... [si se adoptase el pago] esta circunstancia fomentaría i robustecería desde los bancos esa desigualdad entre las clases sociales que la lei debe empeñarse siempre por destruir... el hijo del pobre no puede pagar porque su padre nada tiene [si se adoptase el pago y se exceptuase solo al pobre] el hijo del pobre recibiría la instrucción como una limosna; el hijo del rico como una mercancía" (Amunátegui y Amunátegui, 1856, p. 128).
} 
nueva dinámica importa comprender, tal como lo señalamos en el párrafo anterior, si estamos o no en presencia de una nueva doxa -un nuevo relato respecto de lo educativo- que nos indica el sentido común del mismo: ¿para qué es la educación?; ¿qué podemos esperar de ella?, pero también y sobre todo si existe una nueva illusio en el campo educativo chileno: ¿por qué es importante la disputa en torno a lo educativo?; ¿por qué agentes tan diversos de la sociedad chilena tienen interés en el debate educativo?; ¡ cuál es la recompensa a nivel de reconocimiento e imposición simbólica producto de disputar el campo educativo?

\section{EL DESPLAZAMIENTO}

El hecho de constituirse como escenario simbólico y más aún al reforzarse como mercado educacional sumado al proceso creciente de masificación, hace que la educación, antiguo reducto de las élites dominantes, sea sometida a procesos particulares de diferenciación en la cual se producen fenómenos evidentes y otros sutiles de desplazamiento. En efecto y a diferencia del consumo cultural de otros bienes, los grupos de élite o dominantes de la sociedad no pueden renunciar a la educación, cambiándola por otro bien y por lo mismo aprovecharán el espacio simbólico de la misma para crear sucesivas distinciones que especifican cada vez más el bien educativo (tipos de colegios, tipos de universidades, tipos de carreras, todo ello asociado a parámetros jerárquicos, es decir, de construcción de simbolismos de superioridad e inferioridad social). Con ello, las conquistas sociales reflejadas en la democratización de la educación son devaluadas desde los códigos mayormente asumidos (mediante la posesión y uso de un determinado capital cultural) de quienes dominan el campo. Piénsese por ejemplo en el fenómeno de los liceos secundarios en Chile, instituciones públicas a las cuales asistían con orgullo y como sinónimo de distinción las clases altas del país ${ }^{14}$ hasta que, producto de la masificación educativa, llegaron otros sectores sociales, primero los sectores medios que no fueron disonantes

\footnotetext{
${ }^{14}$ Amanda Labarca se refiere con claridad a este tema al describir el origen de los liceos femeninos en Santiago hacia finales del siglo XIX: "Tanto este Liceo [se refiere al No 1 de Niñas], como el No 2 de Santiago, se destinaban a instruir a las jóvenes de la clase pudiente. La Junta de Vigilancia que asesoraba a la dirección tenía, entre otras facultades, la de ser celosa cancerbera de sus aulas, admitiendo a ellas solo a las hijas de abolengo... Aristocratizados y germanizados, los dos primeros Liceos de Niñas de Santiago, cerraban sistemáticamente sus puertas a las jóvenes de clase media y pobre" (Labarca,1939, pp. 201-202).
} 
mientras fueron minoría pero luego crecieron dando espacio también a la llegada de los sectores populares a este nivel educativo ${ }^{15}$. La respuesta del campo, dominado por los dominantes, es la consagración de distinción, el desplazamiento que ellos realizan a otros espacios educativos para recibir educación secundaria, al punto que, al cabo de un siglo, la palabra misma de liceo sufre, en Chile, una profunda devaluación. Pero, y en esto nos sirve también Bourdieu, no todo son sonrisas y sensaciones de alivio entre los dominantes, en este desplazamiento, en esta fuga, ellos también han perdido. De hecho en el caso del liceo republicano perdieron un bien gratuito que les otorgaba distinción y ahora deben pagar por él bajo la forma de colegios privados ${ }^{16}$. Perdieron, algunos de ellos, espacios de calidad educativa, la tan querida tradición, el prestigio, y la identificación con la República, todos ellos bienes simbólicos que una educación provista a partir de relaciones mercantiles no puede suplir fácilmente a menos que la illusio y la doxa del campo también cambien.

\section{A MANERA DE CONCLUSIÓN}

Una lectura superficial y antojadiza del pensamiento de Bourdieu diría que sus conceptos son inútiles para entender el panorama educacional chileno. En efecto, por un lado se puede concluir que un fenómeno como la reproducción de desigualdades escolares es demasiado evidente como para utilizar las sutilezas de los análisis simbólicos. Por otro, se argumentará que las temáticas relevantes son otras, que el derrotero de la reflexión educativa está claro y que el dilema es cómo llegar a una educación eficaz para todos. Sin embargo, frente a la primera visión, el pensamiento de Bourdieu es interesante en el Chile actual para insinuar que la inequidad educacional si bien se relaciona con el capital económico y cultural, reflejado en diferen-

\footnotetext{
${ }^{15}$ La misma Amanda Labarca relata este proceso de democratización y popularización creciente de los liceos de hombres, también hacia fines del S. XIX: "Los liceos no estaban conectados con la enseñanza primaria común, pues desde 1880 mantenían oficialmente sus preparatorias especiales. Ello no constituyó, sin embargo, un obstáculo serio para las clases populares, porque las exigencias de ingreso a 1 primer año de Humanidades eran tan bajas que abrían fácil acceso a1 niño inteligente. El hecho de que los liceos fiscales fueran absolutamente gratuitos, disminuyó su tradicional tendencia selectiva” (Labarca, 1939, p. 214).

${ }_{16}$ Tema al que alude con absoluta claridad la misma Amanda Labarca: "En realidad, la preferencia no se acentuó en los planteles del Estado, sino en los establecimientos particulares que halagaron a la clientela aristocratizante, respondiendo a sus demandas de exclusividad" (Labarca, 1939, p. 214).
} 
cias abismantes de aprendizajes, también es un tema que se explica por la posesión y distribución del capital simbólico al interior del sistema educacional y que la mera redistribución de los recursos materiales no será capaz de modificar sustantivamente tal desigualdad. Frente a la segunda visión -aquella que indica que la reflexión educacional es sólo o principalmente estándares, resultados de aprendizaje y producción de capital humano- el pensamiento de Bourdieu sirve para recordar que plantearlo de esa manera es sólo un estado particular del campo y del predominio de una doxa y una illusio particular que, no está demás recordarlo, en algún momento fueron imposiciones simbólicas que han pasado a ser consideradas como representación de lo único posible y evidente.

\section{REFERENCIAS}

Amunátegui, M.L. y Amunátegui, G. V. (1856). De la instrucción primaria en Chile: lo que es, lo que debería ser. Santiago: Imprenta del Ferrocarril.

Bourdieu, P. (1970). La reproduction. Paris: Seuil. (1979). La distinction. Paris: Seuil. (1989). La noblesse d’ Etat. Grandes écoles et esprit de corps. Paris: Editions de Minuit. (2000). Les structures sociales de l'économie. Paris: Seuil. (2002). Campo de poder, campo intelectual. Buenos Aires: Montressor Jungla Simbólica. (2007). Razones prácticas. Sobre la teoría de la acción. Barcelona: Anagrama.

Carrasco, A (2008). "Investigación en efectividad y mejora escolar: ¿Nueva agenda?". Revista Iberoamericana sobre Calidad, Eficacia y Cambio en Educación Vol. 6, № 4, pp. 6-23.

Corvalán, J. (2003). "La modalidad de co-pago o financiamiento compartido en la educación subvencionada chilena. Apuntes a partir de los resultados de una investigación". Persona y Sociedad, Vol. XVII, No 1.

Dubet, F. y Martuccelli, D. (1998). En la escuela. Barcelona: Losada.

Fligstein, N. y Dauter, L. (2007). “The sociology of markets”. Annu. Rev. Sociol. 33: $105-28$.

Labarca, A. (1939), Historia de la enseñanza en Chile. Santiago: Publicaciones de la Universidad de Chile.

OCDE (2004). Revisión de políticas nacionales en educación: Chile. París y Santiago: OCDE - MINEDUC.

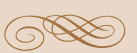

